\title{
IsomiR expression patterns in canonical and Dicer-independent microRNAs
}

\author{
TINGMING LIANG ${ }^{1}$, JIAFENG YU ${ }^{2}$, CHANG LIU ${ }^{1}$ and LI GUO ${ }^{3}$ \\ ${ }^{1}$ Jiangsu Key Laboratory for Molecular and Medical Biotechnology, College of Life Science, Nanjing Normal University, \\ Nanjing, Jiangsu 210023; ${ }^{2}$ Shandong Provincial Key Laboratory of Functional Macromolecular Biophysics, \\ Institute of Biophysics, Dezhou University, Dezhou, Shandong 253023; ${ }^{3}$ Department of Bioinformatics, \\ School of Geographic and Biologic Information, Nanjing University of Posts and Telecommunications,
}

Nanjing, Jiangsu 210023, P.R. China

Received November 5, 2015; Accepted November 11, 2016

DOI: $10.3892 / \mathrm{mmr} .2017 .6117$

\begin{abstract}
Multiple microRNA (miRNA) variants, known as isomiRs, are extensively distributed in miRNA loci and predominantly derive from the alternative cleavage of Drosha/Dicer and 3'addition events. The present study aimed to investigate the expression patterns of multiple isomiRs in typical miRNA and Dicer-independent miRNA loci by conducting evolutionary and expression analysis using public datasets. Although different miRNA maturation processes exist, multiple isomiRs can be detected by similar expression distributions. However, isomiR expression in Dicer-independent miRNA loci tends to be at a moderate level, particularly for random distribution in the ends that are split by Dicer in the typical miRNA loci. Compared with the mature miRNA locus (dominant miRNA locus), the non-dominant miRNA locus indicates an expression distribution similar to that of the Dicer-independent miRNA locus. These results increase the understanding of multiple isomiRs in the progression of diseases.
\end{abstract}

\section{Introduction}

MicroRNAs (miRNAs) are a class of small non-coding RNAs that have been extensively studied as crucial negative regulatory molecules. Canonical miRNA is first processed from a primary miRNA (pri-miRNA) transcript via the Drosha-dependent microprocessor complex (1), and the process generates a precursor miRNA (pre-miRNA) with a stem-loop hairpin structure. Then, it is recognized and cut by Dicer into miRNA-miRNA* duplexes (2) and

Correspondence to: Dr Li Guo, Department of Bioinformatics, School of Geographic and Biologic Information, Nanjing University of Posts and Telecommunications, 9 Wenyuan Road, Qixia, Nanjing, Jiangsu 210023, P.R. China

E-mail:1guo@njupt.edu.cn

Key words: microRNA, isomiR, expression, evolution subsequently pre-miRNA is transported to the cytoplasm by exportin-5 (3). The Dicer protein is widely distributed in plants, metazoans and fungi $(4,5)$, and it is also involved in the processing of other small RNA species, including small nuclear RNAs (6). Recurrent somatic mutations in Drosha can induce changes in miRNA expression (7), and somatic mutations in Drosha and Dicerl can impair miRNA biogenesis (8). Simultaneously, alternative pathways of miRNA biogenesis have been reported (9), and some miRNAs are generated by a novel processing pathway independent of Dicer (10-13). For example, mirtrons can be processed by splicing from precursor hairpins $(14,15)$, and their precursor sequences are shorter than canonical pri-miRNAs because these comprise miRNA-miRNA* duplexes alone. Pre-miRNA hairpins of miRNAs from mirtron-like sources are generated via splicing of short introns and exosome-mediated trimming $(16,17)$.

Typical miRNA biogenesis indicates that mature miRNAs can be incorporated into the RNA-induced silencing complex that then binds to the 3'-untranslated region of the target mRNA to degrade mRNAs or to repress translation (18), while another strand, termed miRNA star (miRNA*), is degraded to an inactive strand. However, accumulating evidence suggests that degraded miRNA* may also serve an important role in gene regulation at the post-transcriptional levels, as well as in the mature miRNA sequence (19-23), and miRNA-miRNA* duplexes are also termed miRNA (miR)-\#-5p-miR-\#-3p duplexes. In the miRNA locus, a series of miRNA variants, called isomiRs, have been widely detected based on high-throughput sequencing datasets (24-30). These multiple isomiRs are predominantly derived from the alternative and imprecise cleavage of Drosha and Dicer during pri-miRNA/pre-miRNA processing, and 3 ' addition events in miRNA maturation processes (27). In the specific miRNA locus, isomiR profiles are always stable across different samples and different species $(26,30)$, indicating a relatively stable miRNA maturation process.

Drosha and Dicer have important roles in the miRNA maturation process, and their imprecise and alternative cleavage processes largely contribute to the generation of multiple isomiRs. It is known that the isomiR profiles are always stable because of Drosha and Dicer, even across 
different animal species, however fewer studies have focused on isomiR expression profiles between typical miRNA loci and Dicer-independent miRNA loci. Generally, several dominant isomiRs (typically one to three) can be identified in the canonical miRNA locus, and others always possess lower expression rates $(26,29,31)$. Therefore, according to characteristics of cleavage and expression patterns of multiple isomiRs, the current study attempted to explore the potential associations between isomiR profiles in different miRNA loci with diversity in the maturation processes, and simultaneously discuss evolutionary patterns of Drosha/Dicer. According to miRNA expression profiles, abundantly expressed miR-451 has been identified as an miRNA that is Dicer-independent, and based on previous studies focusing on isomiR expression (32-34), relevant expression and evolutionary analysis were performed using public datasets. The present study may provide additional information regarding miRNA/isomiR biogenesis.

\section{Materials and methods}

Source data. Both Drosha and Dicer nucleotide and protein sequences of eight vertebrates, including Amphibia (Xenopus tropicalis), Aves (Gallus gallus; Taeniopygia guttata), Mammalia (Homo sapiens; Sus scrofa; Mus musculus), Pisces (Danio rerio) and Sauria (Anolis carolinensis) were collected from GenBank (Table I). Simultaneously, available expression data of miRNA/isomiR in breast cancer $(B C)$ samples $(n=683)$ and normal samples $(n=87)$ were acquired from the Cancer Genome Atlas pilot project (http://cancergenome.nih.gov). Then, due to the fact that the selected BC samples were derived from female patients, samples from other human diseases from the Cancer Genome Atlas pilot project (http://cancergenome. nih.gov) were selected: Thyroid carcinoma (tumor, $n=507$; normal, $n=59$ ) and prostate adenocarcinoma (tumor, $n=498$; normal, $n=52$ ). The selection generated more information about isomiR expression with potential gender differences.

Sequence and expression analyses. The amino acid sequences of Drosha and Dicer were aligned using Clustal X 2.0 (http://www.clustal.org/clustal2) (35), and phylogenetic associations were reconstructed using the MEGA 5.10 software (http://www.megasoftware.net) (36) based on the neighbor-joining method. Genetic distance was simultaneously estimated using MEGA. Functional domains of Drosha and Dicer were identified using the Pfam database (http://pfam. xfam.org) (37), and inferred domains were predicted using SMART (http://smart.embl-heidelberg.de) (38).

According to biological characteristics of isomiRs, the relative expression rate of each isomiR was estimated based on all the isomiRs in the miRNA locus, and then the average expression rate was calculated across different individuals. Herein, due to the larger differences in sample sizes between tumor and normal samples, expression patterns were first estimated using different sample sizes, and then were estimated using equal sample sizes by randomly selecting 87 tumor samples in BC. Simultaneously, relevant analysis was further performed using other samples. According to miRNA/isomiR expression profiles and typical isomiR expression patterns, the simultaneously dominantly expressed miR-\#-5p and miR-\#-3p from hsa-miR-21, -30a and -30e were collected, and another miRNA that was Dicer-independent (miR-451) was selected for further analysis.

Statistical analysis. The expression levels of the isomiRs in multiple individuals, particularly those of dominant isomiRs, were expressed as the mean \pm standard deviation. Differences in genetic distance were estimated using the $t$-test between Drosha and Dicer, and $\mathrm{P}<0.05$ was considered to indicate a statistically significant difference. Simultaneously, the $95 \%$ confidence interval was also estimated. The degree of variation of relative expression of isomiRs was presented using a box plot. Relevant statistical description or analysis was conducted using Stata software, version 11 (StataCorp LP, College Station, TX, USA).

\section{Results and discussion}

The typical miRNA maturation process depends on Drosha and Dicer, and the miRNA-\#-5p:miRNA-\#-3p duplex is generated through cleavage of pri-miRNA and pre-miRNA (Fig. 1A). Although single miRNA sequences have been widely studied, the miRNA locus generates a series of isomiRs with diverse sequences and expression levels that are predominantly derived from the imprecise and alternative cleavage of Drosha/Dicer and 3' addition events (24-30). As a specific sequence in multiple isomiRs, the canonical miRNA sequence is not always the most dominantly expressed sequence (such as hsa-miR-30a-5p in the miRNA locus; Fig. 1) (29). Although sample sizes differed between tumor and normal samples, similar expression patterns were obtained using equally sized samples (Fig. 1). The isomiR expression profiles were always stable across different samples, and this feature was also observed in different cells, tissues and animal species, although abnormal isomiR expression patterns were also detected, particularly in certain diseased samples. The current study attempted to compare isomiR expression profiles between typical miRNA loci and miRNA loci with independent of Dicer using public sequencing datasets. Compared with recent studies on the expression and evolutionary patterns of isomiRs $(29,39-41)$, the present study examined the expression profile of a specific miRNA gene (hsa-miR-30a), that can generate two kinds of mature miRNAs, and a dominantly expressed miRNA independent of Dicer (hsa-miR-451), which has been updated as miR-451a, and was selected due to the fact that fewer miRNAs independent of Dicer are dominantly expressed.

As crucial factors that lead to multiple isomiRs in miRNA maturation, Drosha and Dicer are well-conserved and have similar evolutionary rates across different vertebrates (Fig. 2 and Table II). These similar evolutionary patterns may contribute to recognition and cleavage of Drosha and Dicer in the miRNA maturation process, and further contribute to stable isomiR expression profiles, particularly for the majority of isomiRs that are $3^{\prime}$ isomiRs with the same $5^{\prime}$ ends. In pre-miRNA with a stem-loop structure, dominantly expressed miRNA may be located in the 5 p or $3 p$ arm, however all the miRNA loci generate dominant $3^{\prime}$ isomiRs and rare 5' isomiRs. Notably, more pre-miRNAs have been identified to generate mature and functional miR-\#-5p and miR-\#-3p. 
Table I. Drosha and Dicer in eight vertebrates.

\begin{tabular}{|c|c|c|c|c|}
\hline Species & Abbreviation & Gene & Accession number & Length (AA) \\
\hline Amphibia: Xenopus tropicalis & $\mathrm{xtr}$ & Drosha & NP_001107152.1 & 1,325 \\
\hline Aves: Gallus gallus & gga & Drosha & NP_001006379.1 & 1,336 \\
\hline Aves: Taeniopygia guttata & $\operatorname{tgu}$ & Drosha & XP_002199233 & 1,338 \\
\hline Mammalia: Homo sapiens & hsa & Drosha & Q9NRR4 & 1,374 \\
\hline Mammalia: Sus scrofa & ssc & Drosha & XP_005672458 & 1,373 \\
\hline Mammalia: Mus musculus & $\mathrm{mmu}$ & Drosha & Q5HZJ0 & 1,373 \\
\hline Pisces: Danio rerio & dre & Drosha & NP_001103942 & 1,289 \\
\hline Sauria: Anolis carolinensis & aca & Drosha & XP_003226799 & 1,339 \\
\hline Amphibia: Xenopus tropicalis & $\mathrm{xtr}$ & Dicer & NP_001123390.2 & 1,893 \\
\hline Aves: Gallus gallus & gga & Dicer & NP_001035555.1 & 1,921 \\
\hline Aves: Taeniopygia guttata & $\operatorname{tgu}$ & Dicer & NP_001156875 & 1,921 \\
\hline Mammalia: Homo sapiens & hsa & Dicer & NP_001278557.1 & $1,922^{\mathrm{a}}$ \\
\hline Mammalia: Sus scrofa & $\mathrm{ssc}$ & Dicer & NP_001184123.1 & 1,915 \\
\hline Mammalia: Mus musculus & $\mathrm{mmu}$ & Dicer & NP_683750.2 & 1,906 \\
\hline Pisces: Danio rerio & dre & Dicer & NP_001154925.1 & 1,865 \\
\hline Sauria: Anolis carolinensis & aca & Dicer & XP_003214365 & 1,918 \\
\hline
\end{tabular}

${ }^{a}$ Indicates that Dicer isoforms have been reported in humans, and in the present study only the longer Dicer gene is analyzed.

Table II. Evolutionary distance between Drosha and Dicer.

\begin{tabular}{|c|c|c|c|c|c|c|c|c|}
\hline & hsa & $\mathrm{ssc}$ & $\mathrm{mmu}$ & gga & $\operatorname{tgu}$ & aca & $\mathrm{xtr}$ & dre \\
\hline hsa & - & 0.048 & 0.055 & 0.066 & 0.068 & 0.091 & 0.150 & 0.200 \\
\hline ssc & 0.012 & - & 0.071 & 0.089 & 0.088 & 0.106 & 0.162 & 0.201 \\
\hline $\mathrm{mmu}$ & 0.033 & 0.036 & - & 0.089 & 0.090 & 0.112 & 0.165 & 0.220 \\
\hline gga & 0.112 & 0.111 & 0.115 & - & 0.019 & 0.071 & 0.134 & 0.199 \\
\hline $\operatorname{tgu}$ & 0.121 & 0.119 & 0.127 & 0.045 & - & 0.070 & 0.138 & 0.200 \\
\hline aca & 0.126 & 0.122 & 0.132 & 0.095 & 0.109 & - & 0.144 & 0.198 \\
\hline $\mathrm{xtr}$ & 0.180 & 0.178 & 0.178 & 0.160 & 0.170 & 0.167 & - & 0.213 \\
\hline dre & 0.204 & 0.203 & 0.208 & 0.212 & 0.226 & 0.226 & 0.242 & - \\
\hline
\end{tabular}

Above the diagonal, pairwise genetic distance based on Dicer; below the diagonal, pairwise genetic distance based on Drosha. The $95 \% \mathrm{CI}$ of distance in Drosha was 0.118-0.166, and the 95\% CI of distance in Dicer was 0.101-0.146. No significant difference of genetic distance was detected between Drosha and Dicer ( $\mathrm{t}=1.132, \mathrm{P}=0.263)$. CI, confidence interval.

These observations suggest the relative precise cleavage of Drosha and Dicer on the 5' ends during miRNA maturation process, although they serve important roles in different regions in cells. Divergence of $3^{\prime}$ ends among isomiRs from a given miRNA locus and among miRNAs in different species may be predominantly derived from the imprecise cleavage of Drosha and Dicer on $3^{\prime}$ ends, in addition to modification events following miRNA maturation.

However, compared with typical miRNAs that are generated by cleavage of Drosha and Dicer, certain miRNAs are generated by alternative pathways during miRNA biogenesis (9), including a novel processing pathway independent of Dicer (10-12). For example, miR-451 is generated via the Dicer-independent pathway, and then contributes to the regulatory network as a functional miRNA (13). Although different maturation processes occur in $\mathrm{miR}-451$ and canonical miRNA loci (including miR-21, 30a and 30e), multiple isomiR sequences are also present in the Dicer-independent miRNA locus (Figs. 3-5 and Table III). Similar to typical miRNA loci $(26,29,42)$, several dominant isomiRs are dominantly expressed, and the majority of isomiRs are 3 ' isomiRs. The highly conserved 5 ' ends ensure the consistency of the function between different isomiR sequences, which in turn facilitate the co-regulation of target mRNAs. However, the most dominant isomiR only possesses moderate expression (approximately $45 \%$ of the total expression), and other dominant isomiRs have similar expression levels (Fig. 3A). The moderate expression patterns differ from the most typical miRNAs that are prone to have predominant isomiRs with absolute abundant expression (such as isomiRs from the miR-30a-5p locus). 
Table III. Dominantly expressed isomiR sequences in Figs. 2 and 3.

\begin{tabular}{|c|c|c|}
\hline miRNA & IsomiR species & Sequence \\
\hline \multirow[t]{3}{*}{$\operatorname{miR}-451$} & The most dominant & AAACCGUUACCAUUACUGAGUU \\
\hline & The second dominant & AAACCGUUACCAUUACUGAGU \\
\hline & The third dominant & AAACCGUUACCAUUACUGAGUUU \\
\hline \multirow[t]{3}{*}{$\operatorname{miR}-30 a-5 p$} & The most dominant & UGUAAACAUCCUCGACUGGAAGC \\
\hline & The second dominant & UGUAAACAUCCUCGACUGGAAGCU \\
\hline & The third dominant & UGUAAACAUCCUCGACUGGAAG \\
\hline \multirow[t]{3}{*}{$\operatorname{miR}-30 a-3 p$} & The most dominant & CUUUCAGUCGGAUGUUUGCAGCU \\
\hline & The second dominant & CUUUCAGUCGGAUGUUUGCAGC \\
\hline & The third dominant & UUUCAGUCGGAUGUUUGCAGCU \\
\hline \multirow[t]{3}{*}{ miR-21-5p } & The most dominant & UAGCUUAUCAGACUGAUGUUGAC \\
\hline & The second dominant & UAGCUUAUCAGACUGAUGUUGA \\
\hline & The third dominant & UAGCUUAUCAGACUGAUGUUGACU \\
\hline \multirow[t]{3}{*}{ miR-21-3p } & The most dominant & CAACACCAGUCGAUGGGCUGUCU \\
\hline & The second dominant & CAACACCAGUCGAUGGGCUGUC \\
\hline & The third dominant & CAACACCAGUCGAUGGGCUGU \\
\hline \multirow[t]{3}{*}{$\operatorname{miR}-30 e-5 p$} & The most dominant & UGUAAACAUCCUUGACUGGA \\
\hline & The second dominant & UGUAAACAUCCUUGACUGGAAGC \\
\hline & The third dominant & UGUAAACAUCCUUGACUGGAAGCU \\
\hline \multirow[t]{3}{*}{$\operatorname{miR}-30 e-3 p$} & The most dominant & CUUUCAGUCGGAUGUUUACAGCG \\
\hline & The second dominant & CUUUCAGUCGGAUGUUUACAGC \\
\hline & The third dominant & CUUUCAGUCGGAUGUUUACAG \\
\hline
\end{tabular}

miR, microRNA.

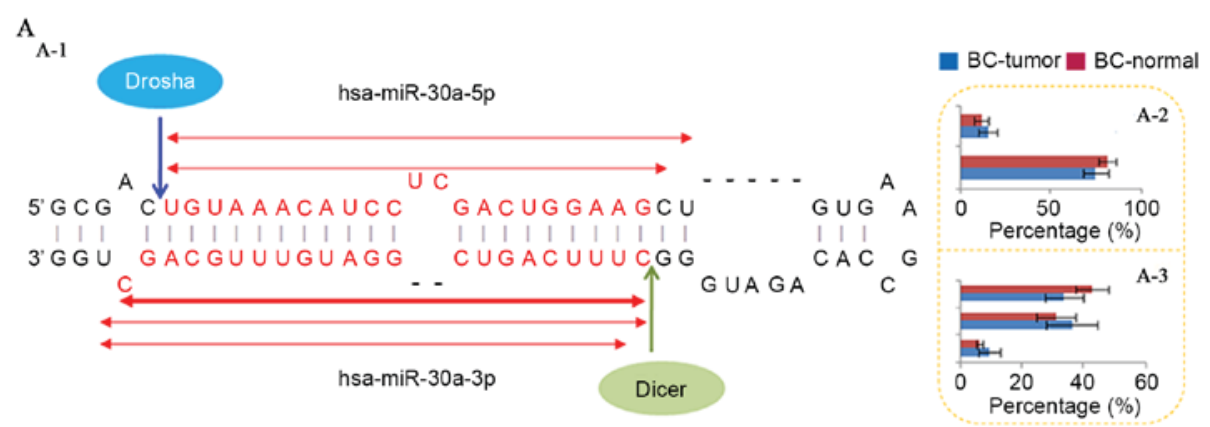

B
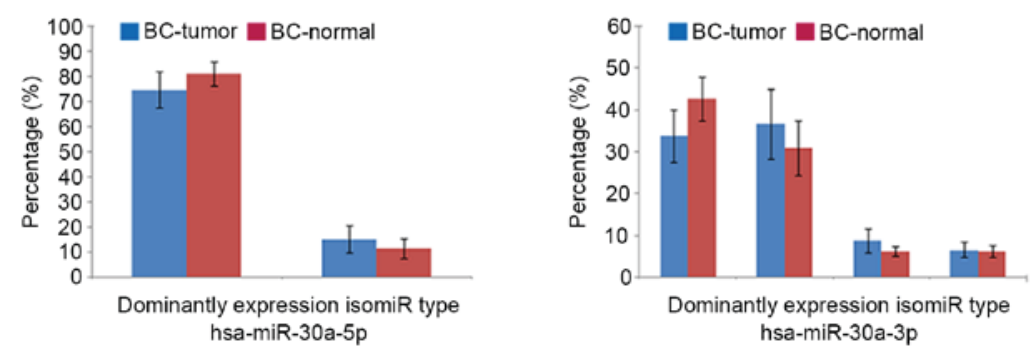

Figure 1. An example of multiple isomiRs and their expression patterns. Percentages (\%) indicate the percentage of a certain isomiR compared with all detected isomiRs in the miRNA locus. (A) Hsa-mir-30a yields miR-30a-5p and miR-30a-3p with a series of multiple isomiRs via cleavage of Drosha and Dicer. Of these, miR-30a-5p is designated as the mature miRNA, whereas miR-30a-3p was termed the miRNA* sequence. (A-1) Red arrows indicate the annotated canonical miR-30a-3p sequence in the miRBase database, whereas the canonical miR-30a-5p sequence is not dominantly expressed. Only dominantly expressed isomiRs are presented here. Expression patterns from the two miRNA loci are presented in (A-2) and (A-3) using samples of breast cancer. (A-2) Expression distribution of two isomiRs in miR-30a-5p; and (A-3) the expression distribution of three isomiRs in miR-30a-3p. In the miR-30a-5p locus, two dominant isomiRs were identified ( $>5 \%$ in the locus), and four dominant isomiRs were detected in the miR-30a-3p locus. The Y axes in Fig. A-2 and A-3 indicate the expression of relevant isomiR sequences in Fig. A-1. (B) Expression patterns were identified for these dominantly expressed isomiRs using tumor and normal samples with the same sample sizes $(\mathrm{n}=87)$. Compared with the expression in $(\mathrm{A})$ in different sample sizes, the expression patterns were considered to be similar. miRNA, microRNAs; BC, breast cancer. 


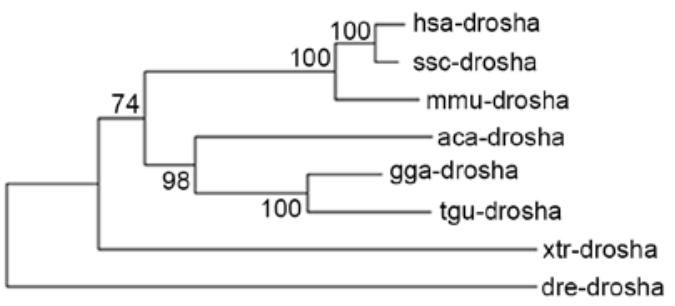

$\stackrel{\longmapsto}{0.02} \quad$ NJ : Drosha

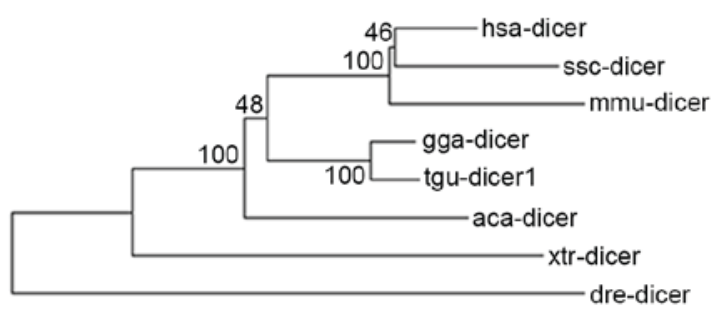

NJ : Dicer

Figure 2. Phylogenetic trees of Drosha and Dicer. Similar phylogenetic associations are detected based on Drosha and Dicer sequences. hsa, Homo sapiens; ssc, Sus scrofa; mmu, Mus musculus; aca, Anolis carolinensis; gga, Gallus gallus; tgu, Taeniopygia guttata; xtr, Xenopus tropicalis; dre, Danio rerio; NJ, neighbor-joining.

A
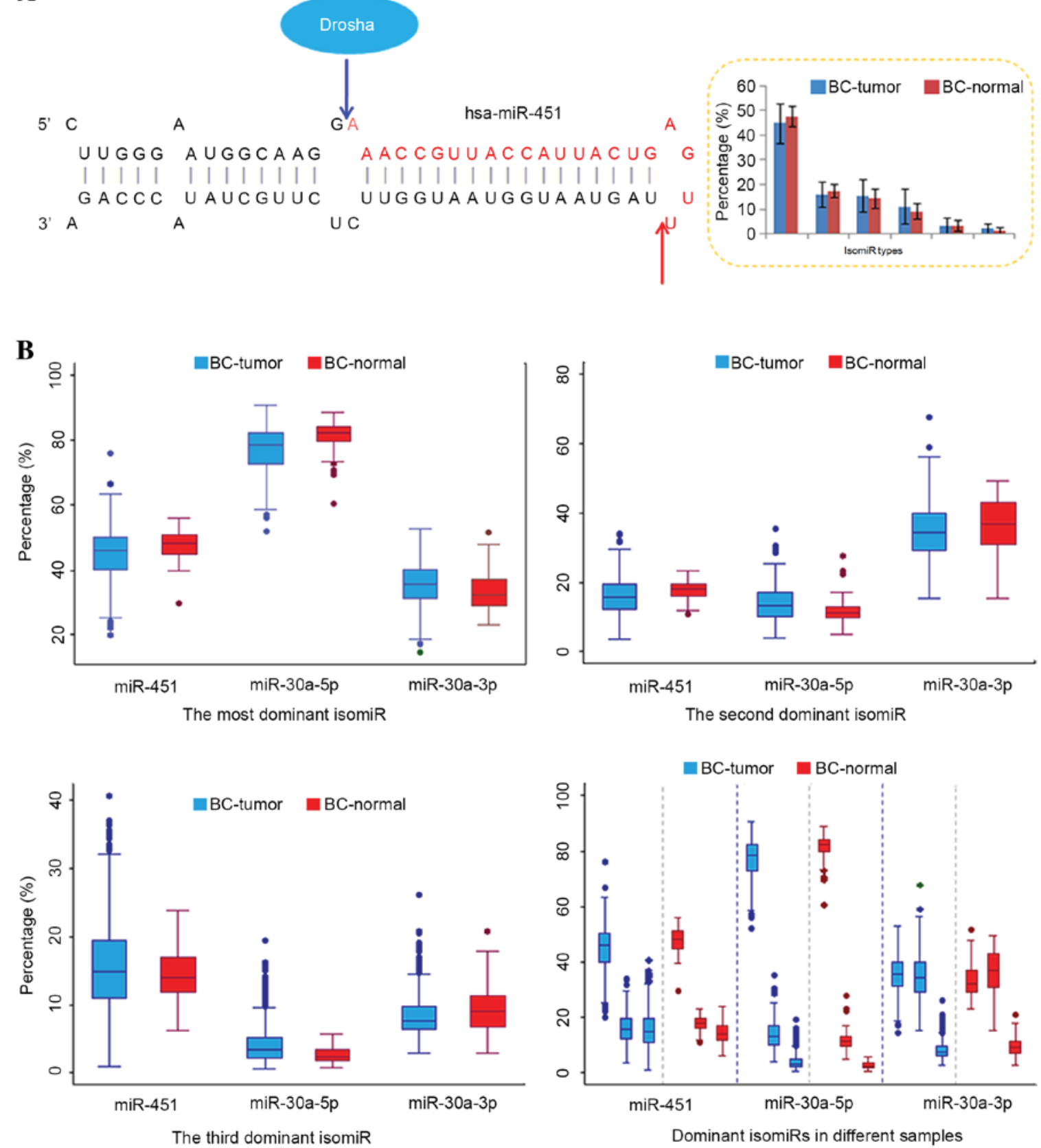

Figure 3. Multiple isomiRs in miR-451 and variations across different samples. Percentage (\%) in the Y-axis indicates the percentage of a certain isomiR compared with all detected isomiRs in the miRNA locus. (A) hsa-miR-451 was identified to undergo Dicer-independent maturation. A similar phenomenon for multiple isomiRs was detected in the miRNA locus. (B) Box plots based on relative expression of isomiRs. According to the most, second and third dominantly expressed isomiRs, isomiRs have a larger degree of variation in tumor samples than normal samples. Compared with miR-30a-5p, the miR-451 locus indicates a moderate level of isomiR expression, which typically involves higher degree of variation in different samples. miR, microRNAs; BC, breast cancer. 

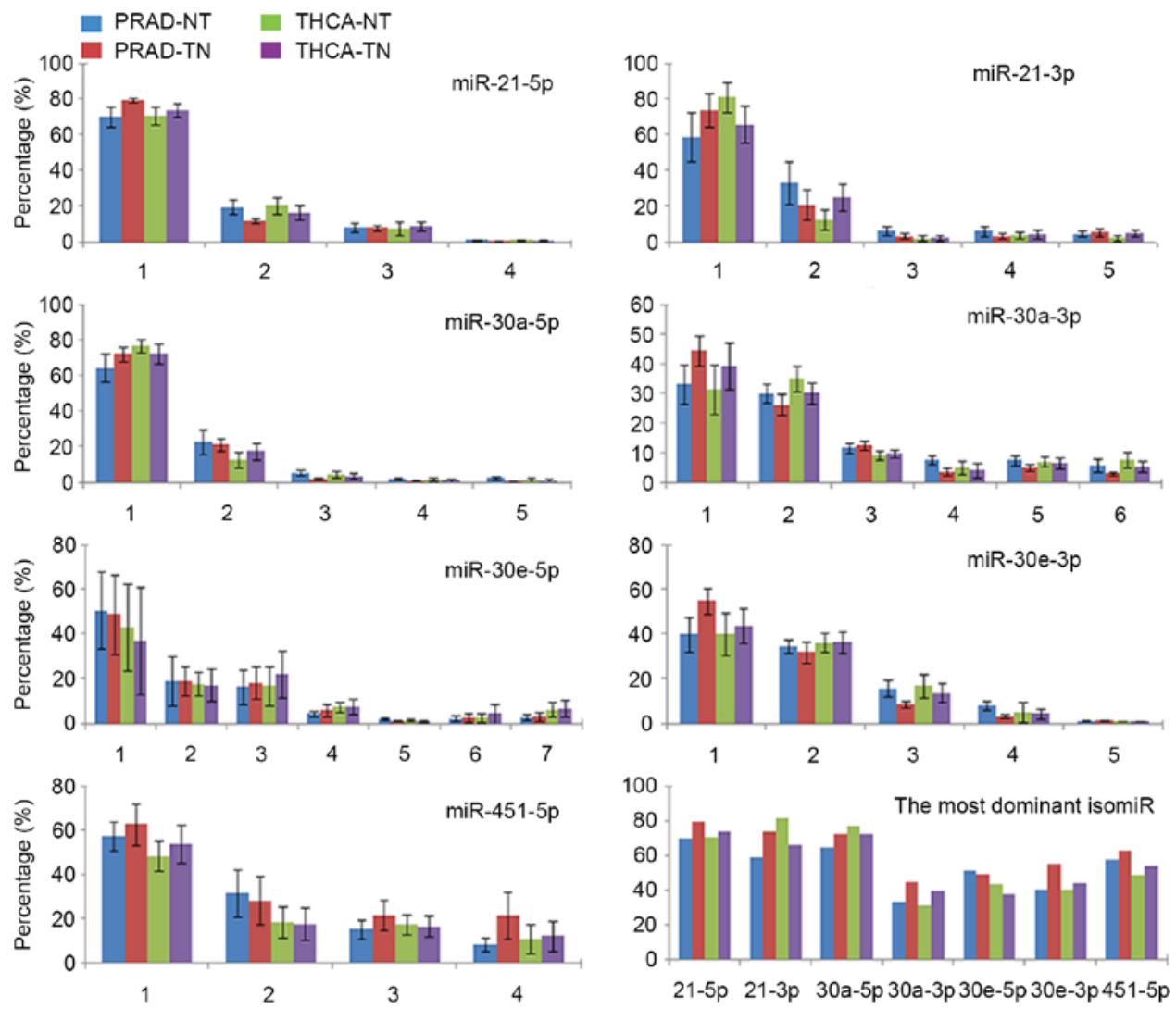

Figure 4. Expression patterns of multiple isomiRs in different miRNA loci and samples. Percentage (\%) in the Y-axis indicates the percentage of a specific isomiR compared with all detected isomiRs in the miRNA locus and the $\mathrm{X}$-axis indicates the isomiR types. A total of three miRNA genes, including miR-21, miR-30a and miR-30e, were selected and analyzed due to the fact that miR-\#-5p and miR-\#-3p were abundantly expressed. Of these, miR-30e-5p was not dominantly expressed (however another strand of miR-30e-3p was upregulated), and miR-451 was also rarely expressed in PRAD and normal samples. Lower expression levels influence the expression pattern of isomiRs. miRNA/miR, microRNA; PRAD, prostate adenocarcinoma; NT, Normal, Matched Tumor; THCA, thyroid carcinoma; TN, Tumor, Matched Normal.
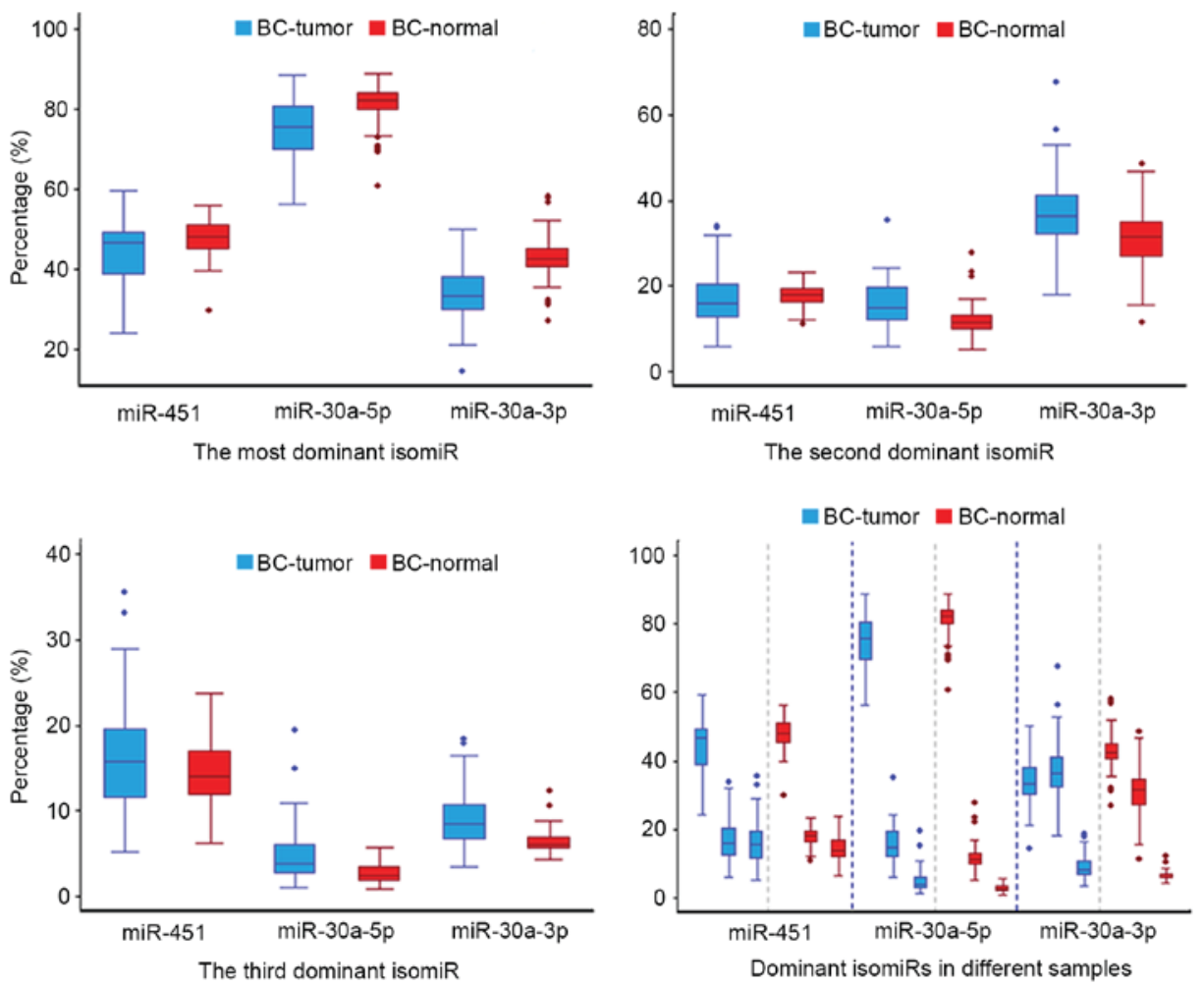

Figure 5. Variations in isomiRs across different samples using equal sample sizes $(\mathrm{n}=87)$. Percentage $(\%)$ in the Y-axis indicates the percentage of a specific isomiR compared with all detected isomiRs in the miRNA locus. miR, microRNA; BC, breast cancer. 
Another non-dominant miRNA strand such as the miR-30a-3p locus induces moderate isomiR expression profiles, similar to that observed in Dicer-independent miR-451 locus (40). Notably, although several dominant isomiRs are present in the passenger strand, distinct differences were detected between dominant isomiRs and other rare isomiRs, whereas in the miR-451 locus, no evident boundary was detected. The moderate level of expression may have resulted from the random cleavage of the hairpin, whereas isomiRs in most typical miRNAs are strictly controlled. The mir-451 hairpin could not be recognized by Dicer because of the insufficient amount of duplexes formed, and therefore it was directly cleaved by Ago2. The Dicer-independent maturation process may lead to relative random cleavage sites at the $3^{\prime}$ ends of miRNAs, which is different from the non-random $3^{\prime}$ ends of multiple isomiRs derived from typical miRNA locus. Generally, in typical miRNA loci, dominant isomiRs are evident, and there are always one to three types of abundantly expressed isomiRs that can be used to infer dominant cleavage sites in Drosha and Dicer. These observations indicate the cleavage bias of Drosha and Dicer, which further contributes to the distinct expression profiles of dominant isomiRs.

The current study also observed that although isomiR expression profiles are always stable across different samples, including between tumor and normal samples, and variations were also detected between diseased and normal samples (39). In diseased samples, a larger degree of variation was observed compared with that among normal samples, which implicates that the miRNA maturation process may be affected in the abnormal micro-environment (Figs. 3 and 5). The dispersed expression patterns of isomiRs may be derived from changes in Drosha and Dicer cleavage, modification or regulation following miRNA maturation in tumor samples. These distinct characteristics may furthermore provide additional information on the dynamic expression profiles of the coding-non-coding RNA regulatory network.

In conclusion, expression analysis of isomiRs demonstrated that Dicer-independent miRNA involves a moderate level of isomiR expression compared with that observed in typical miRNA loci. Notably, a similar expression profile can be detected between non-dominant miRNA and Dicer-independent miRNA loci, thereby suggesting more complex miRNA maturation processes, particularly at the isomiR levels. Dynamic miRNA/isomiR expression profiles further enrich the regulatory network, particularly those involving miRNA-miRNA and miRNA-mRNA interactions. Additional studies on multiple isomiRs, particularly their potential versatile biological roles, may prove beneficial.

\section{Acknowledgements}

The current study was supported by the National Natural Science Foundation of China (grant nos. 31271261, 31401009 and 61301251), the Program for the Top Young Talents by the Organization Department of the CPC Central Committee, the National Natural Science Foundation of Jiangsu (grant no. BK20130885), the Key Project of Social Development in Jiangsu Province (grant no. BE2016773), the Research Fund for the Doctoral Program of Higher Education of China (grant no. 20133234120009), Shandong Provincial Key
Laboratory of Functional Macromolecular Biophysics, the Priority Academic Program Development of Jiangsu Higher Education Institutions (PAPD), sponsored by NUPTSF (grant no. NY215068).

\section{References}

1. Gregory RI, Yan KP, Amuthan G, Chendrimada T, Doratotaj B, Cooch N and Shiekhattar R: The Microprocessor complex mediates the genesis of microRNAs. Nature 432: 235-240, 2004.

2. He L and Hannon GJ: MicroRNAs: Small RNAs with a big role in gene regulation. Nat Rev Genet 5: 522-531, 2004.

3. Lund E, Güttinger S, Calado A, Dahlberg JE and Kutay U: Nuclear export of microRNA precursors. Science 303: 95-98, 2004.

4. Cerutti $\mathrm{H}$ and Casas-Mollano JA: On the origin and functions of RNA-mediated silencing: From protists to man. Curr Genet 50: 81-99, 2006.

5. Shabalina SA and Koonin EV: Origins and evolution of eukaryotic RNA interference. Trends Ecol Evol 23: 578-587, 2008.

6. Langenberger D, Bermudez-Santana CI, Stadler PF and Hoffmann S: Identification and classification of small RNAs in transcriptome sequence data. Pac Symp Biocomput 80-87, 2010.

7. Torrezan GT, Ferreira EN, Nakahata AM, Barros BD, Castro MT, Correa BR, Krepischi AC, Olivieri EH, Cunha IW, Tabori U, et al: Recurrent somatic mutation in DROSHA induces microRNA profile changes in Wilms tumour. Nat Commun 5: 4039, 2014.

8. Rakheja D, Chen KS, Liu Y, Shukla AA, Schmid V, Chang TC, Khokhar S, Wickiser JE, Karandikar NJ, Malter JS, et al: Somatic mutations in DROSHA and DICER1 impair microRNA biogenesis through distinct mechanisms in Wilms tumours. Nat Commun 2: 4802, 2014

9. Yang JS and Lai EC: Alternative miRNA biogenesis pathways and the interpretation of core miRNA pathway mutants. Mol Cell 43: 892-903, 2011.

10. Cheloufi S, Dos Santos CO, Chong MM and Hannon GJ: A dicer-independent miRNA biogenesis pathway that requires Ago catalysis. Nature 465: 584-589, 2010

11. Cifuentes D, Xue H, Taylor DW, Patnode H, Mishima Y, Cheloufi S, Ma E, Mane S, Hannon GJ, Lawson ND, et al: A novel miRNA processing pathway independent of Dicer requires Argonaute2 catalytic activity. Science 328: 1694-1698, 2010.

12. Miyoshi K, Miyoshi T and Siomi H: Many ways to generate microRNA-like small RNAs: Non-canonical pathways for microRNA production. Mol Genet Genomics 284: 95-103, 2010.

13. Yang JS, Maurin T, Robine N, Rasmussen KD, Jeffrey KL, Chandwani R, Papapetrou EP, Sadelain M, O'Carroll D and Lai EC: Conserved vertebrate mir-451 provides a platform for Dicer-independent, Ago2-mediated microRNA biogenesis. Proc Natl Acad Sci USA 107: 15163-15168, 2010.

14. Okamura K, Hagen JW, Duan H, Tyler DM and Lai EC: The mirtron pathway generates microRNA-class regulatory RNAs in Drosophila. Cell 130: 89-100, 2007.

15. Ruby JG, Jan CH and Bartel DP: Intronic microRNA precursors that bypass Drosha processing. Nature 448: 83-86, 2007.

16. Flynt AS, Greimann JC, Chung WJ, Lima CD and Lai EC: MicroRNA biogenesis via splicing and exosome-mediated trimming in Drosophila. Mol Cell 38: 900-907, 2010.

17. Chong MM, Zhang G, Cheloufi S, Neubert TA, Hannon GJ and Littman DR: Canonical and alternate functions of the microRNA biogenesis machinery. Genes Dev 24: 1951-1960, 2010.

18. Kim VN, Han J and Siomi MC: Biogenesis of small RNAs in animals. Nat Rev Mol Cell Bio 10: 126-139, 2009.

19. Okamura K, Phillips MD, Tyler DM, Duan H, Chou YT and Lai EC: The regulatory activity of microRNA* species has substantial influence on microRNA and 3' UTR evolution. Nat Struct Mol Biol 15: 354-363, 2008.

20. Czech B, Zhou R, Erlich Y, Brennecke J, Binari R, Villalta C, Gordon A, Perrimon N and Hannon GJ: Hierarchical rules for Argonaute loading in Drosophila. Mol Cell 36: 445-456, 2009.

21. Okamura K, Liu N and Lai EC: Distinct mechanisms for microRNA strand selection by Drosophila Argonautes. Mol Cell 36: 431-444, 2009.

22. Guo L and Lu Z: The fate of miRNA* strand through evolutionary analysis: Implication for degradation as merely carrier strand or potential regulatory molecule? PLoS One 5: e11387, 2010. 
23. Jagadeeswaran G, Zheng Y, Sumathipala N, Jiang H, Arrese EL, Soulages JL, Zhang W and Sunkar R: Deep sequencing of small RNA libraries reveals dynamic regulation of conserved and novel microRNAs and microRNA-stars during silkworm development. BMC Genomics 11: 52, 2010.

24. Landgraf P, Rusu M, Sheridan R, Sewer A, Iovino N, Aravin A, Pfeffer S, Rice A, Kamphorst AO, Landthaler M, et al: A mammalian microRNA expression atlas based on small RNA library sequencing. Cell 129: 1401-1414, 2007.

25. Morin RD, Aksay G, Dolgosheina E, Ebhardt HA, Magrini V, Mardis ER, Sahinalp SC and Unrau PJ: Comparative analysis of the small RNA transcriptomes of Pinus contorta and Oryza sativa. Genome Res 18: 571-584, 2008.

26. Guo L, Yang Q, Lu J, Li H, Ge Q, Gu W, Bai Y and Lu Z: A comprehensive survey of mirna repertoire and 3' addition events in the placentas of patients with pre-eclampsia from high-throughput sequencing. PLoS One 6: e21072, 2011.

27. Neilsen CT, Goodall GJ and Bracken CP: IsomiRs-the overlooked repertoire in the dynamic microRNAome. Trends Genet 28: 544-549, 2012.

28. Lee LW, Zhang S, Etheridge A, Ma L, Martin D, Galas D and Wang K: Complexity of the microRNA repertoire revealed by next generation sequencing. RNA 16: 2170-2180, 2010.

29. Guo L and Chen F: A Challenge for miRNA: Multiple isomiRs in miRNAomics. Gene 544: 1-7, 2014.

30. Burroughs AM, Ando Y, de Hoon MJ, Tomaru Y, Nishibu T, Ukekawa R, Funakoshi T, Kurokawa T, Suzuki H, Hayashizaki Y and Daub CO: A comprehensive survey of 3' animal miRNA modification events and a possible role for 3 ' adenylation in modulating miRNA targeting effectiveness. Genome Res 20 : 1398-1410, 2010

31. Guo L, Li H, Lu J, Yang Q, Ge Q, Gu W, Bai Y and Lu Z: Tracking miRNA precursor metabolic products and processing sites through completely analyzing high-throughput sequencing data. Mol Biol Rep 39: 2031-2038, 2012.

32. Loher P, Londin ER and Rigoutsos I: Isomir expression profiles in human lymphoblastoid cell lines exhibit population and gender dependencies. Oncotarget 5: 8790-8802, 2014.
33. Llorens F, Hummel M, Pantano L, Pastor X, Vivancos A, Castillo E, Mattlin H, Ferrer A, Ingham M, Noguera M, et al: Microarray and deep sequencing cross-platform analysis of the mirRNome and isomiR variation in response to epidermal growth factor. BMC Genomics 14: 371, 2013.

34. Guo L, Yu J, Liang T and Zou Q: miR-isomiRExp: A web-server for the analysis of expression of miRNA at the miRNA/isomiR levels. Sci Rep 6: 23700, 2016.

35. Larkin MA, Blackshields G, Brown NP, Chenna R, McGettigan PA, McWilliam H, Valentin F, Wallace IM, Wilm A, Lopez R, et al: Clustal W and clustal X version 2.0. Bioinformatics 23: 2947-2948, 2007.

36. Tamura K, Stecher G, Peterson D, Filipski A and Kumar S: MEGA6: Molecular evolutionary genetics analysis version 6.0. Mol Biol Evol 30: 2725-2729, 2013.

37. Finn RD, Bateman A, Clements J, Coggill P, Eberhardt RY, Eddy SR, Heger A, Hetherington K, Holm L, Mistry J, et al: Pfam: The protein families database. Nucleic Acids Res 42: D222-D230, 2014

38. Letunic I, Doerks T and Bork P: SMART: Recent updates, new developments and status in 2015. Nucleic Acids Res 43: 257-260, 2014.

39. Guo L, Yu J, Yu H, Zhao Y, Chen S, Xu C and Chen F: Evolutionary and expression analysis of miR-\#-5p and miR-\#-3p at the miRNAs/isomiRs levels. Biomed Res Int 2015: 168358, 2015.

40. Guo L, Zhang H, Zhao Y, Yang S and Chen F: Selected isomiR expression profiles via arm switching? Gene 533: 149-155, 2014.

41. Guo L, Zhao Y, Zhang H, Yang S and Chen F: Close association between paralogous multiple isomiRs and paralogous/orthologues miRNA sequences implicates dominant sequence selection across various animal species. Gene 527: 624-629, 2013.

42. Guo L, Chen F and Lu Z: Multiple IsomiRs and Diversity of miRNA Sequences Unveil Evolutionary Roles and Functional Relationships Across Animals. MicroRNA and Non-Coding RNA: Technology, Developments and Applications. pp127-144, 2013. 